\title{
Dal Centro Storico alla Città Storica: la dimensione progettuale della conservazione - Il caso di Roma
}

\section{Do Centro Histórico à Cidade Histórica: a dimensão do projeto de conservação - o caso da cidade de Roma}

\author{
Elio TRUSIANI ${ }^{1}$
}

\section{RESUMO}

Con l'adozione del Piano Regolatore, Roma ha finalmente una nuova prospettiva per costruire il suo sviluppo all'interno di un sistema storico-ambientale tutelato e valorizzato, base concreta della sostenibilità, finalizzato alla riorganizzazione funzionale e spaziale delle sue strutture insediative. Una città proiettata in una dimensione metropolitana e organizzata secondo un modello policentrico, in cui si pone all'attenzione degli addetti ai lavori il superamento del vecchio concetto di centro "storico", legato alla parte più antica del centro urbano, e l'assunzione del concetto di "città storica", comprendente un universo più vasto e diffuso nel territorio (dal medioevo al Rinascimento al Novecento). Un metodo innovativo di lettura della città: non più per zone omogenee ma per tessuti- questo permette si superare il modo di trattamento unitario per intere zone e leggerne invece le differenze, soprattutto in una città come Roma, e pertanto leggerne le attuali esigenze di trasformazione, cercando di conferire, o restituire, al termine città storica il difficile compito di regolare la valorizzazione, orientare le strategie di riqualificazione, costruire le condizioni del progetto, recuperando, in tal senso, la dimensione progettuale della conservazione.

Palavras-chave: cittá storica, centro storico, conservazione.

\section{RESUMO}

Com a adoção do Plano Regulador, Roma finalmente adquiriu uma nova perspectiva para construir o seu desenvolvimento, partir de um sistema histórico-ambiental tutelado e valorizado (base concreta da sustentabilidade), valorizando a reorganização funcional e espacial das suas estruturas subterrâneas. Uma cidade projetada em uma dimensão metropolitana e organizada segundo um modelo policêntrico no qual se chama atenção aos trabalhos regulares e superamentos do velho conceito de centro "histórico", conjugado à parte mais antiga do centro urbano, e a assunção do conceito de "cidade histórica" -

1 Arquitecto, doutore em Urbanismo, Professor da Faculdade de Arquitectura da Universidade de Roma "La Sapienza" e da Faculdade de Arquitectura de Ascoli Piceno, da Universidade de Camerino. 
TRUSIANI, E. Dal Centro Storico alla Città Storica...

compreende um universo mais vasto e difuso no território (do medieval ao Renascimento ao Novecentos). Um método inovador de leitura da cidade, não mais por zonas homogêneas mas por tecidos - isso permite superar o modo de tratamento unitário por zonas inteiras e lê-las ao invés de diferenciá-las, sobretudo em uma cidade como Roma, e para tanto ler as atuais exigências de transformação, procurando conferir, ou restituir, ao final cidade histórica a difícil tarefa de regular a valorização, orientar as estratégias de requalificação, construir as condições do projeto, recuperando, em tal sentido, a dimensão projetual da conservação.

Palavras-chave: cidade histórica, centro histórico, conservação.

\section{Il Piano Regolatore di Roma: brevi cenni introduttivi}

Dopo oltre quarant'anni dal Piano Regolatore del 1962, il Comune di Roma, ha adottato il 5 marzo 2003, il nuovo Piano Regolatore. L'iter per la formazione del piano è durato sette anni di studi per approfondire le conoscenze dell'immenso territorio comunale (1300 Kmq) e portare a termine quel processo di pianificazione che aveva visto con il Piano delle Certezze, adottato nel 1997, la prima tappa importante del nuovo processo di piano del Comune di Roma. Il Piano regolatore conclude, quindi, un lavoro iniziato con il Piano delle Certezze: questo prendeva in considerazione e definiva alcuni caratteri strutturali del territorio come la città consolidata, includendo al suo interno il centro storico, le parti esterne da tutelare, mentre, per la città della trasformazione, rimandava ogni decisione al nuovo strumento.

Con l'adozione del piano, Roma ha finalmente una nuova prospettiva per costruire il suo sviluppo all'interno di un sistema storico-ambientale tutelato evalorizzato, base concreta della sostenibilità, finalizzato alla riorganizzazione funzionale e spaziale delle sue strutture insediative. Una città proiettata in una dimensione metropolitana e organizzata secondo un modello policentrico.

Le principali innovazioni introdotte dal nuovo piano urbanistico riguardano prima di tutto, il modello organizzativo del territorio. Il nuovo piano adotta un modello basato su un sistema di centralità diffuse, articolate in due livelli: metropolitano e locale. Le centralità metropolitane (circa venti) sono ubicate in corrispondenza dei nodi dei sistemi di comunicazione, in modo da costituire una rete di relazioni fisiche e immateriali in grado di rispondere alle esigenze della metropoli contemporanea. Queste centralità sono destinate ad accogliere le funzioni e i servizi che la città offre, sia a livello nazionale e internazionale che al territorio della Provincia e della Regione; al contrario, le centralità locali (circa cinquanta) sono destinate agli abitanti di un intorno urbano più limitato e ben definito.

Gli interventi connessi al policentrismo riguardano la riqualificazione della periferia che riguarderà, non solo la realizzazione di infrastrutture e servizi mancanti, ma soprattutto il decentramento di funzioni pregiate (ricerca, università, cultura, ricettività, commercio, oltre alle residenze) all'interno delle nuove centralità metropolitane.

La tutela del territorio si estende per circa 87.000 ettari e la "campagna romana" è considerata non più soltanto come territorio non urbanizzato riservato alla produzione agricola, ma come parte fondamentale di un sistema ambientale complesso da sottoporre, quindi, ad una attenta pianificazione per la salvaguardia e la valorizzazione degli elementi che lo costituiscono.

Un altro elemento innovativo è il metodo di lettura della città, non più per zone omogenee come dettato dal decreto del 1968, ma per tessuti; questo permette si superare il modo di trattamento unitario per intere zone e leggerne invece le differenze, soprattutto in una città come Roma, e pertanto leggerne le attuali esigenze di trasformazione, di riprogettazione di parti della città esistente, soprattutto dove si verificano fenomeni di abbandono o di perdita di qualità.

Questo ha permesso il superamento del vecchio concetto di "centro storico"legato alla parte più antica del centro urbano e l'assunzione del concetto di "città storica" che comprende un universo più vasto e diffuso nel territorio (dal medioevo al Rinascimento al Novecento).

Un ulteriore elemento innovativo riguarda il tentativo di includere nel piano quegli interventi ordinari introdotti dalla normativa nazionale e dai provvedimenti ministeriali degli anni Novanta; questo vuol dire che il piano ingloba, nella sua normativa tecnica di attuazione, gli strumenti facenti capo alla cosiddetta famiglia dei "programmi complessi". 


\section{Dal "centro storico" alla "città storica"}

Si è accennato brevemente al passaggio dal concetto di centro storico a quello di città storica, ${ }^{2}$ questo ha significato superare un concetto difensivo e cristallizzante, per sviluppare una grande e dovuta attenzione alle potenzialità evolutive di un patrimonio qualitativo che solo ora viene colto e assunto in una sua essenza sistemica, anche da individuare selettivamente nella discontinuità e possibilità d'integrazione.

Questa scelta ha riaperto con determinazione un dibattito di vitale importanza quale il rapporto tra conservazione e trasformazione, ovvero tra le due dimensioni intrinseche al concetto di tradizione la cui dialettica è stata motore di evoluzione della forma urbana. E lo si è fatto assumendo nella definizione di città storica, congiuntamente, sia il sistema dei valori sia quello delle funzioni, nel loro reciproco sostanziarsi e in una perfetta integrazione tra quantità e qualità. Un'integrazione ricercata a partire ancora una volta dal centro storico, assunto come polarizzazione urbana sulla quale si accentrano sistemi radiali o radiocentrici, di natura sia storico-morfologica, sia funzionale e dinamica.

Una polarizzazione antica, sopravvissuta al tempo, ora inserita in un nuovo disegno di policentrismo metropolitano del quale può costituire una delle centralità; infatti viene a porsi come centralità delle centralità, sia per essere la concentrazione di sistemi di valori e di funzioni, sia per l'investimento simbolico e psicologico che la tradizione vi ha depositato.

Per non trascurare gli aspetti essenziali della conservazione si è studiato un modello, fondato sull'integrazione di due categorie di intervento: una di tipo regolativo e una di tipo programmatico. La prima è riservata ai tessuti ${ }^{3}$ urbani omogenei, dotati di per sé di una consolidata qualificazione storico-morfologica mentre la seconda è rivolta agli ambiti urbani strategici per l'attivazione di dinamiche anche trasformative.
La dimensione regolativa del piano si basa su una descrizione e regolamentazione della città storica che si affida alla scala intermedia dei tessuti; questa è ritenuto la scala più idonea per consolidare le qualità strutturanti la forma urbana ereditata ma capace al tempo stesso di recuperare a monte la scala delle morfologie insediative e a valle quella del singolo edificio e spazio aperto elementare, anche attraverso una dimensione argomentativa che supporta la certezza delle regole con la flessibilità interpretativa dei possibili percorsi di conoscenza e progetto.

Gli ambiti di programmazione strategica ${ }^{4}$ sintetizzano il risultato della ricerca di una strumentazione progettuale capace di ricondurre a un quadro di coerenza fatti urbani appartenenti a categorie analitiche e normative diverse ma legati da nessi relazionali strutturanti, in riferimento al "doppio sistema" dei valori e delle funzioni. Gli ambiti individuati sono cinque: due hanno un carattere morfogenetico originario e attraversano l'intera città da nord a sud, verso il mare: l'ambito Tevere e quello dell' asse nordsud. Due hanno un carattere strutturale e radiocentrico: l'ambito delle mura e quello della cintura ferroviaria. Infine un ambito è specificatamente archeologico monumentale e ambientale del settore sud-ovest ed è costituito dal sistema dei Fori Imperiali, dall'area archeologica di Caracalla e dall'Appia Antica. Sono cinque tracce di racconti urbani, dense di storie passate e di altre future, auspicabili e possibili, cinque sentieri narrativi che aprono a molteplici riscoperte e prefigurazioni e nei quali la maggior forza di alcuni materiali e di alcune scelte di valorizzazione sollecita modificazioni fisiche, funzionali e di senso dei materiali più deboli.

Il passaggio da centro storico alla città storica, pertanto, non significa omogeneizzare ed appiattire tutto quello che c'è dentro e trattare diversamente ciò che è fuori dal suo perimetro, ma significa riconoscere le differenze che caratterizzano le diverse parti. Saperle descrivere e conservare, e al contempo suggerire dove necessario, le

\footnotetext{
2 Per Città Storica si intende l'insieme integrato costituito dall'area storica centrale interna alle mura, dalle parti urbane dell'espansione otto-novecentesca consolidata interne ed esterne alle mura e dai singoli siti e manufatti localizzati nell'intero territorio comunale che presentano una identità storico-culturale definita da particolari qualità, riconoscibili e riconosciute dal punto di vista deicaratteri morfogenetici e strutturanti dell'impianto urbano e di quelli tipomorfologici, architettonici e d'uso dei singoli tessuti, edifici e spazi aperti, anche in riferimento al senso e al significato da essi assunto nella memoria delle comunità insediate

3 Si intendono per tessuti della Città Storica gli isolati o parti di isolato ad essa appartenenti costituiti dall'aggregazione di edifici, con relativi spazi aperti di pertinenza e l'esclusione delle sedi viarie, riconducibili a regole sostanzialmente omogenee d'impianto, suddivisione del suolo, disposizione e rapporto con i tracciati, nonché di prevalente caratterizzazione tipo-morfologica, figurativa, tecnico-strutturale e funzionale. Rientrano in tali tessuti gli edifici seriali e quelli speciali esprimenti le stesse regole del tessuto di appartenenza.

4 Gli ambiti di programmazione strategica costituiscono le linee strutturanti della città storica, intesa come macro sistema integrato.
} 
TRUSIANI, E. Dal Centro Storico alla Città Storica...

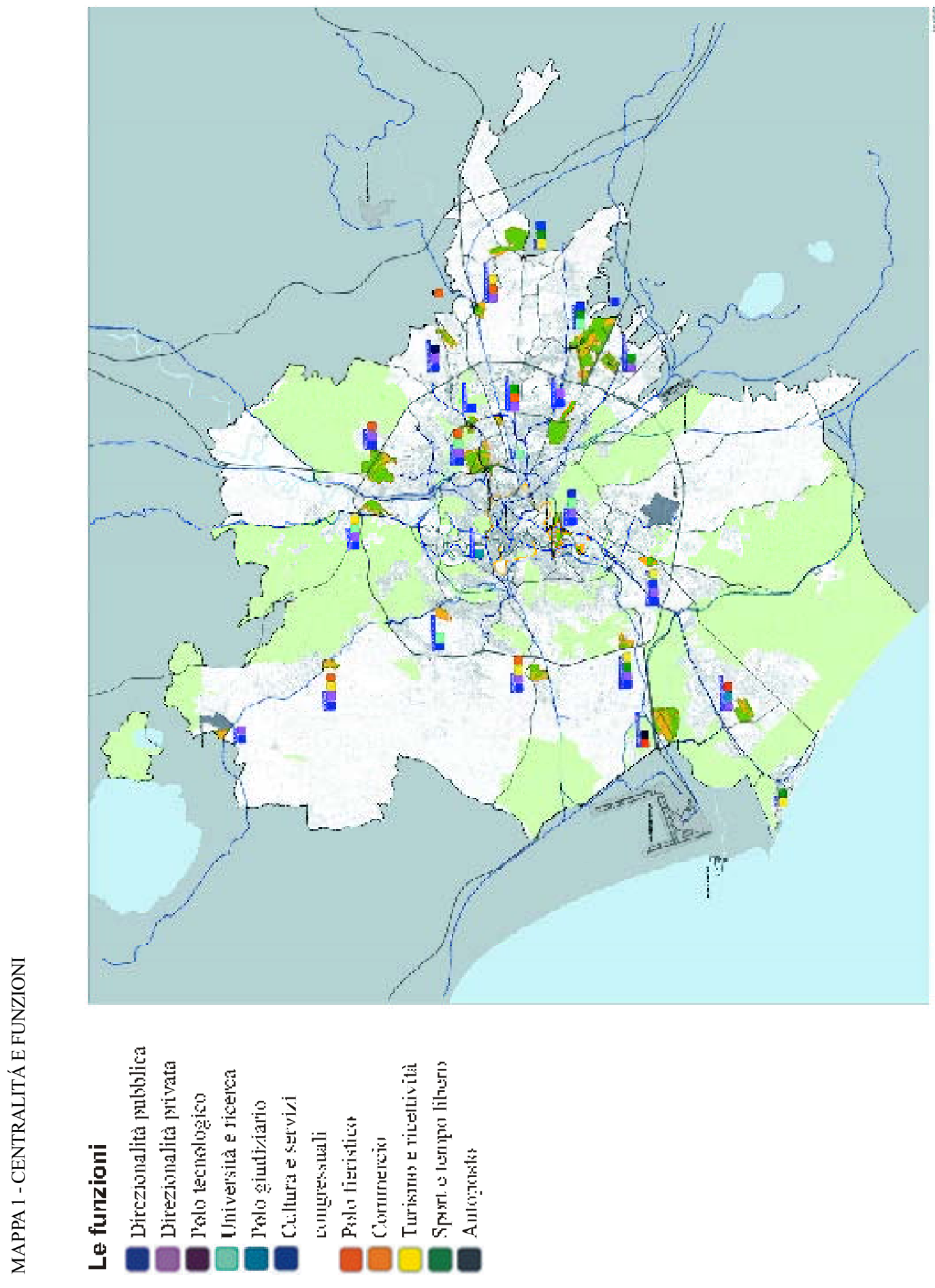


TRUSIANI, E. Dal Centro Storico alla Città Storica...

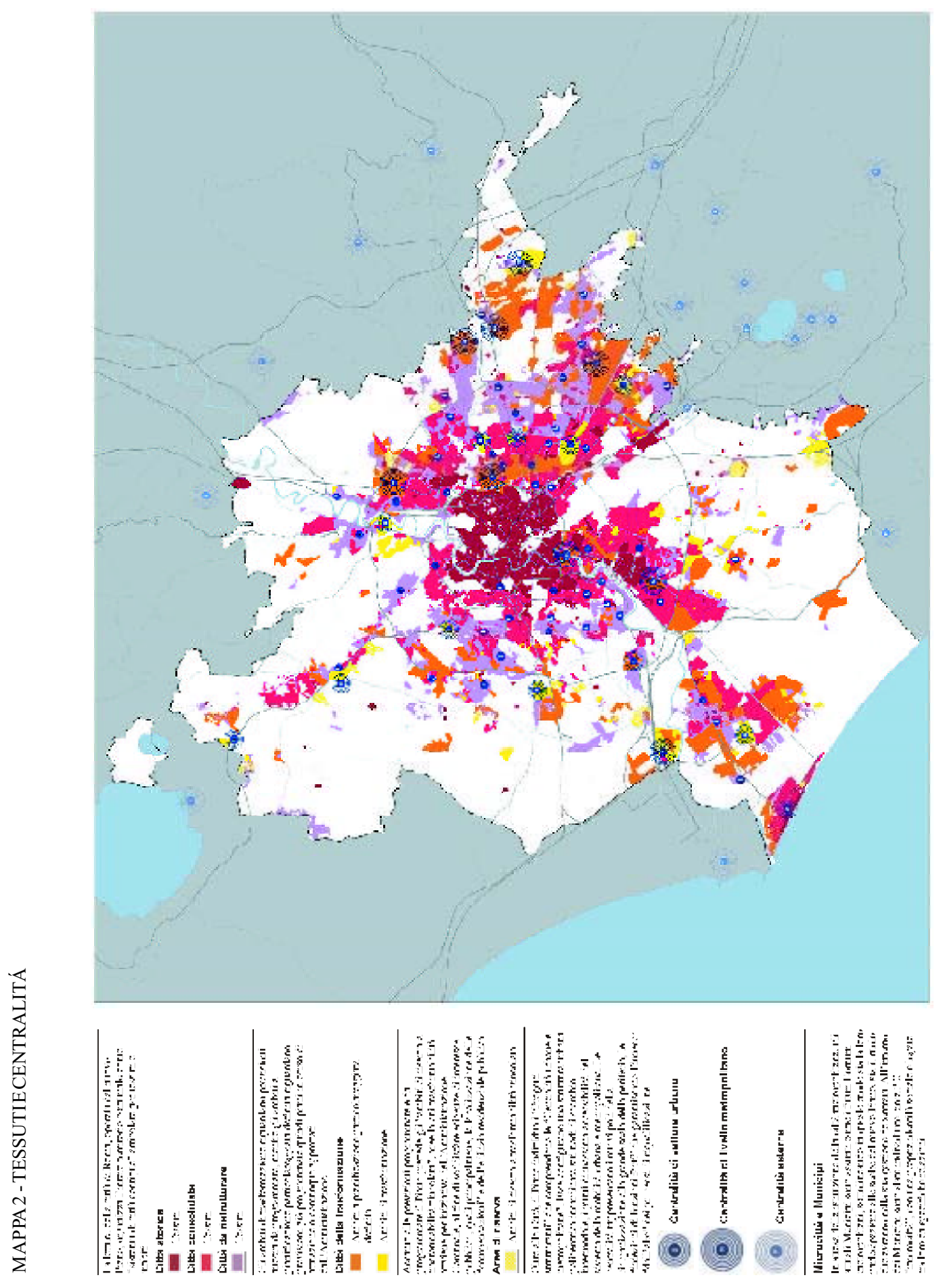

Desenvolvimento e Meio Ambiente, n. 9, p. 93-99, jan.jun. 2004. Editora UFPR 
modificazioni compatibili con quelle differenze. Vuol dire ricercare nuove e strategiche relazioni tra le parti sia all'interno della città storica sia tra questa e la città da consolidare e da trasformare. Questo approccio del piano è il risultato di un'operazione di ricucitura di un processo iniziato molti anni prima, attraverso una molteplicità di studi, ricerche, programmi e progetti di soggetti che negli ultimi anni hanno percorso questa strada e su questa strada hanno iniziato a ragionare. Primo fra tutti il Comune che con le sue esperienze durante le giunte Argan e Petroselli aveva concentrato la propria attenzione sulla città postunitaria, producendo piani e progetti che alludevano a una necessaria estensione del campo anche oltre i confini stretti delle mura avanzando ipotesi di nuovi principi della conservazione e valorizzazione. In tal senso il Piano ha sistematizzato, da un lato, il patrimonio di analisi, studi, ricerche e progetti prodotti in questi anni e, dall'altro, ha operato quel salto verso un processo di consolidamento di nuovi principi della valorizzazione e conservazione. In sostanza ha cercato di conferire, o restituire, al termine città storica il difficile compito di regolare la valorizzazione, orientare le strategie di riqualificazione, costruire le condizioni del progetto.

$\mathrm{Si}$ assiste ad un ripensamento del ruolo della storia negli strumenti urbanistici, e delle modalità attraverso cui un piano debba cambiare per fare i conti non solo con una condizione prescrittiva ma anche con la necessità di raccontare la città e le sue storie passate e future in termini differenti da quelli che la legge prevede. Nel piano di Roma, come accennato precedentemente, convivono due modi di raccontare la città e di governare la sua conservazione e trasformazione che si integrano ma mantengono anche una necessaria e fertile autonomia.

Sia nella dimensione regolativa che in quella strutturale e strategica è insita la consapevolezza del ruolo centrale di una dimensione progettuale della conservazione e valorizzazione della città storica: progetto di conoscenza dei tanti e diversi edifici e spazi aperti $^{5}$ che il progetto implicito del palinsesto storico ci consegna e che la vitalità dei processi ci obbliga a considerare nell'individualità del caso per caso, sebbene all'interno delle coordinate regolative delle descrizioni interpretative prodotte. Al contempo è anche progetto esplicito di modificazione e valorizzazione dei tanti luoghi della città storica che reclamano una riscoperta e una nuova dimensione di senso entro il sistema di relazioni spaziali, percettive, ecologiche e funzionali dei grandi segni della natura e della storia che definiscono la dimensione strutturale e strategica del piano.

Il racconto della città storica avviene per tessuti e spazi aperti, a differenza di quanto fatto precedentemente con il tradizionale colore unitario volto ad indicare la zona " $\mathrm{A}$ " di centro storico; questo lega inscindibilmente le diverse modalità di aggregazione, disposizione e conformazione morfologica, architettonica e costruttiva di edifici e spazi aperti di pertinenza alle differenti fasi storiche di formazione e quindi alle diverse culture dell'abitare e dello spazio urbano, rstituendo alla città storica quella porgetualità in itinere, fondamento stesso della nozione di città.

La dimensione progettuale della conservazione risulta pertanto evidente ed è, al contempo, elemento fondamentale per il raggiungimento della sostenibilità urbanistica,${ }^{6} \mathrm{di}$

5 Sono Spazi aperti della Città storica tutte quelle componenti che costituiscono il sistema dei “vuoti” e che, unitamente con le parti costruite, in ragione della riconoscibilità, della compiutezza storico-morfologico-architettonica e della connotazione dei caratteri orografici ed ecologico-ambientali, partecipano alla definizione dell'identità della città

6 Il concetto di "sostenibilità urbanistica" rappresenta l'applicazione più significativa dell'integrazione tra urbanistica e ecologia, nelle più recenti esperienze di pianificazione in Italia, ed è al contempo un obiettivo esplicito di molti piani dell'ultima generazione nonché una esplicita richiesta delle normative regionali degli ultimi anni. Gli elementi che caratterizzano questa integrazione la sostenibilità urbanistica in genere, modificando in modo sostanziale il modello ambientale precedente, sono sostanzialmente due: il superamento dell'approccio di tutela e conservazione come orizzonte fondamentale del piano urbanistico-ambientale e la compatibilità ambientale del sistema infrastrutturale.

In sintesi la sostenibilità urbanistica si è concretizzata in tre aspetti fondamentali:

a) l'esclusione di nuove forme di espansione urbana, orientando il piano verso la manutenzione qualitativa (recupero e completamento) e la trasformazione della città esistente, evitando il più possibile un ulteriore consumo di suolo extraurbano;

b) la compatibilità ambientale ed ecologica del sistema infrastrutturale, con nuove soluzioni per la mobilità urbana che privilegino il trasporto urbano collettivo e la riduzione degli impatti (rumore, paesaggio) delle infrastrutture della mobilità mentre per quanto riguarda le reti tecnologiche, la relativa compatibilità ambientale comporta la separazione delle reti bianche e nere della fognatura, la raccolta e lo smaltimento diretto delle acque piovane nel suolo permeabile per evitare il sovraccarico del sistema fognario, l'esclusione di nuovi insediamenti nelle zone urbane sprovviste di efficienti reti idriche e fognarie, etc.

c) l'applicazione dei principi della rigenerazione ambientale in tutti gli interventi di attuazione del piano, con opere di ripermeabilizzazione dei suoli urbani più impermeabilizzati, legati alla manutenzione della città esistente e alla trasformazione urbanistica; la diffusione massiccia del verde privato, l'aumento di quello pubblico, la garanzia di elevate quote di superfici permeabili nei nuovi insediamenti, la creazione di una rete ecologiche colleghi gli elementi ambientali, naturali e artificiali, presenti all'esterno e all'interno della città. 
TRUSIANI, E. Dal Centro Storico alla Città Storica...

cui il sistema del patrimonio storico-ambientale è parte importante se non determinante; infatti sono proprio le regole della gestione del patrimonio edilizio esistente ( $\mathrm{i}$ tessuti) e della trasformazione urbanistica (ambiti e centralità): i nuovi indici e parametri urbanistici ed ecologici riducono sensibilmente il carico urbanistico rispetto alle modalità con cui è stata realizzata la città moderna densità, altezze, etc) e la regola generalizzata del mix funzionale garantisce un ambiente urbano sempre vivibile e mai segregato. Si afferma il principio che anche la trasformazione urbanistica, cioè l'oggetto principale del piano urbanistico di oggi, e non solo la conservazione, può garantire il miglioramento delle condizioni ecologiche della città, nell'ottica di uno sviluppo sostenibile, e che questo miglioramento può essere valutato in termini oggettivi, attraverso un bilancio qualitativo delle risorse ambientali fondamentali interessate dalla trasformazione, oltre che rispetto alla disponibilità e accessibilità delle aree relative. Non solo standard quindi, ma una migliore qualità dell'aria, l'equilibrio del ciclo dell' acqua, la bonifica e risanamento dei suoli inquinati, il rafforzamento della biodiversità sono i portati di una trasformazione urbanistica che incide concretamente sulla qualità dell'ambiente.

\section{Brevi considerazioni a margine}

In sostanza il Piano individua nella politica della tutela e della salvaguardia, nonché nel principio della conservazione, un'azione non solo passiva di regime puramente vincolistico, ma fortemente innovativa in termini di proposizione e di approccio alla modificazione e trasformazione, riconoscendo, nel sistema delle differenze, al valore della storia e alla storia della città, intesa come processo di stratificazione di fatti urbani e di usi ed azioni comportamentali, il valore di risorsa primaria per una progettazione sostenibile dell'ambiente urbano, naturale e fisico. Pertanto la tutela e la salvaguardia, non più intese solo come limite al nuovo ma come stimolo per il nuovo e fondamento per quei processi urbani di consolidamento o, semplicemente, adeguamento della città secondo quel processo in atto che muove dal centro storico alla città esistente, ed ancora, al territorio storico. Un processo, ed un percorso culturale, in cui il riconoscimento dei valori del patrimonio storico viene assunto come punto di partenza per il progetto della città, storica e contemporanea, ove il concetto di conservazione e recupero è inteso anche come trasformazione, riproponendo l'importanza del progetto di città secondo un approccio di più ampio respiro, che sappia cogliere, leggere ed interpretare i cambiamenti in divenire e coniugarli con le reali esigenze di trasformazione e sostenibilità urbanistica. 